\title{
Typhlodromips swirskii (Acari: Phytoseiidae): A Predator of Eriophyid and Tetranychid Mango Mites in Egypt
}

\author{
B. A. Abou-Awad ${ }^{*}$ A. M. Metwally ${ }^{* * *}$ and M. M. Al-Azzazy ${ }^{* *}$ \\ *Plant Protection Dept., National Research Center, 12622 Dokki, Cairo, Egypt \\ ${ }^{* *}$ Agricultural Zoology and Nematology Dept., Faculty of Agric., Al- Azhar Univ., Cairo
}

\begin{abstract}
The predacious mite Typhlodromips swirskii (Athias-Henriot) successfully developed to the adult stage when fed on the motile stages of the mango bud mite Aceria mangiferae Sayed, the mango rust mite Metaculus mangiferae (Attiah), the leaf coating mite Cisaberoptus kenyae Keifer and nymphs of the mango red mite Oligonychus mangiferus (Rahman and Sabra) in the laboratory at different temperatures and relative humidities. The increase of temperature degree and decrease relative humidity from $25^{\circ} \mathrm{C}$ and $60 \%$ R.H. to $30^{\circ} \mathrm{C} \& 55 \%$ R.H. and $35{ }^{\circ} \mathrm{C} \& 50 \%$ R.H. shortened development and increased reproduction and prey consumption. Different eriophyid prey species promoted faster development of the predator compared to feeding on the tetranychid nymphs. The rate of egg laying $(2.62,2.23,2.35$ and $1.83 \mathrm{eggs} /$ $/$ day) was recorded at the highest temperatures and lowest R.H., while the minimum reproduction (1.92, $1.72,1.62$ and $1.20 \mathrm{eggs} /$ / /day) was noted at the lowest temperature and highest R.H. when fed on the four aforementioned prey species, respectively. Life table parameters indicated that feeding T. swirskii on A. mangiferae led to the highest reproduction rate $(\mathrm{rm}=0.216$ and 0.157 females/female/day), while feeding on $O$. mangiferus gave the lowest reproduction rate ( $\mathrm{rm}=0.183$ and 0.133 ) nymphs at $35^{\circ} \mathrm{C}$ and $50 \%$ R.H. and $25^{\circ} \mathrm{C}$ and $60 \%$ R.H., respectively. The adult predatory female consumed an average of $117.8 \mathrm{~A}$. mangiferare, $114.18 \mathrm{C}$ kenyae, $94.4 \mathrm{M}$. mangiferae motile stages and 14.58 O. mangiferus nymphs at $35^{\circ} \mathrm{C}$ and $50 \%$ R.H./day, while it devoured 102.8, $96.59,90.44$ and 12.35 individuals, respectively at $25^{\circ} \mathrm{C}$ and $60 \%$ R.H. The three eriophyid mango mites, particularly A. mangiferae, proved to be suitable prey for $T$. swirskii, as a facultative predator, compared to the tetranychid mango mite.
\end{abstract}

Key Words: Typhlodrompis swirskii, Phytoseiidae, Eriophyidae, Tetranychidae.

\section{INTRODUCTION}

Typhlodromips swirskii (Athias-Henriot) (= Amblyseius swirskii Athias-Henriot) has a significant role in the biological control of some mite pests in Egypt (Yousef and Shehata, 1971; Momen and El-Sawi, 1993; Abou-Awad et al., 1999). It feeds not only on phytophagous mites, but also on coccids and mealy bugs (Swirski et al., 1967; Ragusa and Swirski, 1976; Metwally et al., 1984). During a 2-year study on abandoned mango trees near Cairo, three specific eriophyid mango, i.e. Mango bud mite Aceria mangiferae Sayed, Mango rust mite Metaculus mangiferae (Attiah) and Leaf coating mite Cisaberoptus kenyae Keifer and the tetranychid. Mango red mite Oligonychus mangiferus (Rahman and Sabra) were noted associated with $85 \%$ of the samples. Their populations started to increase in May and reached their peaks in August, then tailed off in December (Al-Azzazy, 2005).

Many phytoseiids have low rates of egg production below 50\% R.H. Low humidity affects development and predatory efficiency of phytoseiids (Sabelis, 1985; van Dinh et al., 1988; Mangini and Hain, 1991; Abou-Elela, 2003). The present study reveals the effect of different temperature degrees and relative humidities on the development of $T$. swirskii on mango mite pests. Special attention was also paid to the effect of eriophyid and tetranychid mites infesting mango trees on the life table parameters of the predator.

\section{MATERIALS AND METHODS}

The efficiency of $T$. swirskii as a predator was studied in the laboratory at different temperature degrees and relative humidities, i.e. $25 \pm 1^{\circ} \mathrm{C} \& 60 \%$ R.H.; $30 \pm 1^{\circ} \mathrm{C} \& 55 \%$ R.H.; $35 \pm 1^{\circ} \mathrm{C} \& 50 \%$ R.H. and a $12 / 12 \mathrm{~h}$ light/dark period, against motile stages of the mango bud mite $A$. mangiferae, the mango rust mite $M$. mangiferae, the leaf coating mite $C$. kenyae and nymphs of the mango red mite $O$. mangiferus. Individuals of $T$. swirskii were obtained from a mass culture maintained on the eriophyid mite Eriophyes olivi Zaher and Abou-Awad. Gravid females were left for $24 \mathrm{~h}$ to lay eggs. Eggs were then isolated for the different biological tests. Mulberry leaf discs, Morus alba L., $2.0 \mathrm{~cm}$ in diameter, were used as rearing arenas in Petri dishes with upper surfaces downwards on water saturated cotton wool.

Predatory eggs were placed singly on individual arenas, and the newly hatched larvae, 50 for every test, were supplied with a food resource of each of the four aforementioned prey. Due to the difficulty of transferring the eriophyid bud mite $A$. mangiferae and the two eriophyids mango leaves $M$. mangiferae 
and C. kenyae, an outer bract of heavily infested bud or a small disc $0.25 \mathrm{~cm}$ in diameter of heavily infested host leaves was carefully examined and the total number of individuals per each was recorded before introduced them to the arenas. Replacement of the consumed prey was carried out daily and notes on development, food consumption and reproduction were recorded twice a day. After the last moulting, males were coupled with females for mating. Males were then transferred to new arenas and individually reared until death. Every 3-4 days, the predators were transferred to new arenas, while its eggs were removed daily from the arenas. To test the sex ratio, 30 eggs were confined, singly in new arenas and the hatched larvae were reared until maturity. Life table parameters were estimated according to Hulting et al., (1990).

\section{RESULTS AND DISCUSSION}

The present results revealed that increase of temperature from $25{ }^{\circ} \mathrm{C}$ to $35{ }^{\circ} \mathrm{C}$ joined with a decrease in relative humidity from $60 \%$ to $50 \%$ enhanced faster development of $T$. swirsikii, and adult longevity was significantly shortened. The four prey mite species, i.e. A. mangifeae, M. mangiferae, $C$. Kenya and $O$. mangiferus resulted in a similar trend (Tables $1 \& 2$ ).

It is also of interest to note that increasing the temperature degree up to $35^{\circ} \mathrm{C}$ joined with decreasing the relative humidity enhanced a higher rate of egg laying. Moreover, feeding the predator on the eriophyid mite A. mangiferae resulted in the highest female fecundity followed by that of females fed on the other two eriophyid species $M$. mangiferae and C. Kenyae. On the other hand, feeding the predator on the tetranychid mite $O$. mangiferus gave in the lowest fecundity (Table 3).

However, these results are in agreement with that reported by Abou- Awad et al., (1999) when reared the predator on the fig bud mite Aceria ficus (Colte) and the fig leaf mite, Rhyncaphytoptus ficifoliae Keifer. Also similar results were reported by El-Laithy and Fouly (1992) when reared the predator on the two spotted spider mite Tetranychus urticae Koch and that of Metwally et al., (1984) who reared the predator on the citrus brown mite Eutetranychus orientalis Klien.

It is worth noting that feeding the predator on each of the four prey did not result in a significant effect on the rate of development of the different stages (Tables $1 \& 2$ ).
In the feeding activity experiment, it was noted that the eriophyid mite $A$. mangiferae was the most favourable prey to the predator followed by the other two eriophyid prey species. On the other hand, the tetranychid prey $O$. mangiferus was the least suitable prey compared with the other aforementioned prey (Tables 3-7).

The preference of the eriophyid mites as prey for the phytoseiid mites compared to the tetranychid prey was reported by several workers (Abou-Awad et .al., 1989; Momen and El-Sawy, 1993; Momen, 1999, Rasmy et al., 2002) and Abou-Awad et al., 2005.

In addition, the daily rate of feeding capacity of the predatory females positively increased with increasing temperature degree joint with decreasing humidity, whereas the predatory immatures displayed opposite trend.

Life table parameters presented in table 7 are in harmony with the aforementioned findings. The population of $T$. swirskii could multiply with (23.82, 24.94 and 32.81), (19.58, 22.51 and 25.71), (22.26, 23.10 and 27.47) and (14.62, 14.34 and 18.47) net reproduction rate within a generation time of (20.10, 18.31 and 16.16 days), (20.52, 17.90 and 16.01), (21.20, 17.29 and 16.92) and (20.14, 17.05 and 15.87) when the predator fed on A. mangiferae, M. mangiferae, C. kenyae and O.mangiferus at $25 \&$ $60 ; 30 \& 55$ and $35{ }^{\circ} \mathrm{C} \& 50 \%$ R.H., respectively. Under these conditions, feeding T. swirskii on A. mangiferae led to the highest reproduction rate $(\mathrm{rm}=0.157,0.175$ and 0.216 females/female/day $)$, while feeding on $O$. mangiferus resulted in the lowest reproduction rate $(0.133,0.156$ and 0.183$)$. It is worth noting that the sex ratio of the progeny of females fed on motile stages of different eriophyid preys favoured females compared with feeding on O. mangiferus.

Comparing the life table parameters of $T$. swirskii with those of other workers carried out under almost similar conditions showed that the net reproduction rate (Ro) and the mean generation time $(\mathrm{T})$ were 13, 17 on T. urticae (El-Laithy and Fouly, 1992) and 16, 17 on E. orientalis (Aly, 1994), respectively; but on the fig bud mite $A$. ficus, the predator population could multiply 21 times in a generation time of 20 days (Abou-Awad et al., 1999). Here again, eriophyid mites proved to be more suitable prey for phytoseiid mites than tetranychids.

This study provides a basic biological 
Table (1): Average of developmental durations in days of the immature stages of the predatory phytoseiid mite Typhlodromips swirskii fed on four mite species at different temperatures and relative humidities.

\begin{tabular}{|c|c|c|c|c|c|c|}
\hline Temperature $\left({ }^{\circ} \mathrm{C}\right) \&$ R. $\mathrm{H}$. & Sex & Egg & Larva & Protonymph & Deutonymph & Life cycle \\
\hline \multicolumn{7}{|l|}{ A. mangiferae } \\
\hline \multirow{2}{*}{$25 \pm 1$ and $60 \%$} & 9 & $i .08 \pm 0.39$ & $.85 \pm 0.12$ & $2.43 \pm 0.24$ & $2.51 \pm 0.24$ & $9.87 \pm 0.60 \mathrm{a}$ \\
\hline & $\hat{\sigma}$ & $\therefore .76 \pm 0.24$ & $.45 \pm 0.09$ & $2.07 \pm 0.23$ & $2.09 \pm 0.20$ & $8.37 \pm 0.54 \mathrm{a}$ \\
\hline \multirow{2}{*}{$30 \pm 1$ and $55 \%$} & o & $\therefore .92 \pm 0.01$ & $.26 \pm 0.21$ & $2.03 \pm 0.24$ & $2.03 \pm 0.20$ & $8.24 \pm 0.24 b$ \\
\hline & 0 & $\therefore .50 \pm 0.20$ & $.26 \pm 0.24$ & $1.84 \pm 0.17$ & $1.57 \pm 0.18$ & $7.17 \pm 0.39 b$ \\
\hline \multirow{2}{*}{$35 \pm 1$ and $50 \%$} & 우 & $. .36 \pm 0.35$ & $.15 \pm 0.00$ & $1.34 \pm 0.09$ & $1.35 \pm 0.02$ & $6.20 \pm 0.53 c$ \\
\hline & 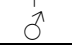 & $\therefore .16 \pm 0.24$ & $.13 \pm 0.00$ & $1.14 \pm 0.00$ & $1.12 \pm 0.0$ & $5.55 \pm 0.20 \mathrm{c}$ \\
\hline \multicolumn{7}{|l|}{ M. mangiferae } \\
\hline \multirow{2}{*}{$25 \pm 1$ and $60 \%$} & q & $i .09 \pm 0.35$ & $.52 \pm 0.24$ & $2.42 \pm 0.20$ & $2.59 \pm 0.09$ & $9.62 \pm 0.23 a$ \\
\hline & $\hat{0}$ & $\therefore .93 \pm 0.27$ & $.34 \pm 0.20$ & $2.28 \pm 0.24$ & $2.42 \pm 0.20$ & $8.97 \pm 0.20 \mathrm{a}$ \\
\hline \multirow{2}{*}{$30 \pm 1$ and $55 \%$} & 우 & $3.0 \pm 0.24$ & $.50 \pm 0.07$ & $1.63 \pm 0.17$ & $1.87 \pm 0.09$ & $8.00 \pm 0.17 \mathrm{~b}$ \\
\hline & $\hat{0}$ & $\therefore 69 \pm 0.20$ & $.42 \pm 0.19$ & $1.88 \pm 0.20$ & $1.80 \pm 0.08$ & $7.79 \pm 0.19 b$ \\
\hline \multirow{2}{*}{$35 \pm 1$ and $50 \%$} & 우 & $\therefore .50 \pm 0.19$ & $.25 \pm 0.01$ & $1.25 \pm 0.03$ & $1.18 \pm 0.00$ & $6.18 \pm 0.29 c$ \\
\hline & $\sigma^{+}$ & $\therefore 46 \pm 0.23$ & $.15 \pm 0.00$ & $1.14 \pm 0.00$ & $1.15 \pm 0.00$ & $5.90 \pm 0.33 c$ \\
\hline \multicolumn{7}{|l|}{ C. kenyae } \\
\hline \multirow{2}{*}{$25 \pm 1$ and $60 \%$} & 우 & $3 \pm 0.01$ & $.32 \pm 0.02$ & $2.23 \pm 0.00$ & $2.24 \pm 0.00$ & $8.79 \pm 0.67 \mathrm{a}$ \\
\hline & $\hat{0}$ & $\therefore .86 \pm 0.24$ & $.22 \pm 0.00$ & $2.15 \pm 0.19$ & $2.22 \pm 0.00$ & $8.45 \pm 0.44 \mathrm{a}$ \\
\hline \multirow{2}{*}{$30 \pm 1$ and $55 \%$} & q & $\therefore .60 \pm 0.18$ & $.41 \pm 0.07$ & $2.01 \pm 0.02$ & $2.11 \pm 0.18$ & $8.13 \pm 0.39 b$ \\
\hline & o & $\therefore .57 \pm 0.27$ & $.25 \pm 0.09$ & $1.75 \pm 0.20$ & $1.75 \pm 0.20$ & $7.30 \pm 0.41 b$ \\
\hline \multirow{2}{*}{$35 \pm 1$ and $50 \%$} & 우 & $\therefore .41 \pm 0.20$ & $.24 \pm 0.08$ & $1.32 \pm 0.20$ & $1.42 \pm 0.17$ & $6.39 \pm 0.56 c$ \\
\hline & $\hat{\sigma}^{+}$ & $. .25 \pm 0.24$ & $1.13 \pm 0.0$ & $1.21 \pm 0.17$ & $1.21 \pm 0.07$ & $5.80 \pm 0.49 \mathrm{c}$ \\
\hline \multicolumn{7}{|l|}{ O. mangiferus } \\
\hline \multirow{2}{*}{$25 \pm 1$ and $60 \%$} & q & $.30 \pm 0.11$ & $.36 \pm 0.07$ & $2.27 \pm 0.00$ & $2.46 \pm 0.20$ & $9.39 \pm 0.74 \mathrm{a}$ \\
\hline & $\hat{\sigma}^{+}$ & $.16 \pm 0.20$ & $30 \pm 0.019$ & $2.23 \pm 0.00$ & $2.31 \pm 0.36$ & $9.00 \pm 0.44 \mathrm{a}$ \\
\hline \multirow{2}{*}{$30 \pm 1$ and $55 \%$} & 우 & $\therefore .83 \pm 0.24$ & $.26 \pm 0.00$ & $2.08 \pm 0.01$ & $2.16 \pm 0.09$ & $8.33 \pm 0.33 b$ \\
\hline & o & $\therefore .60 \pm 0.24$ & $.21 \pm 0.00$ & $1.86 \pm 0.02$ & $2.13 \pm 0.20$ & $7.80 \pm 0.65 b$ \\
\hline \multirow{2}{*}{$35 \pm 1$ and $50 \%$} & 우 & $\therefore .40 \pm 0.20$ & $.17 \pm 0.00$ & $1.27 \pm 0.01$ & $1.27 \pm 0.03$ & $6.11 \pm 0.27 \mathrm{c}$ \\
\hline & $\hat{0}$ & $\therefore 27 \pm 0.17$ & $.14 \pm 0.00$ & $1.14 \pm 0.00$ & $1.14 \pm 0.00$ & $5.69 \pm 0.33 c$ \\
\hline
\end{tabular}

Mean \pm SD: Different letters in vertical columns denote significant difference (F- test, $\mathrm{P}<0.05, \mathrm{P}<0.01)$.

Table (2): Average durations in days of the phytoseiid mite Typhlodromips swirskii adults fed on different mite prey at different temperatures and relative humidities.

\begin{tabular}{|c|c|c|c|c|c|c|c|c|}
\hline \multirow{2}{*}{$\begin{array}{c}\text { Temperature }\left({ }^{\circ} \mathrm{C}\right) \\
\text { \& R.H. }\end{array}$} & \multirow{2}{*}{$\begin{array}{c}\text { Pre - } \\
\text { Oviposition }\end{array}$} & \multirow{2}{*}{ Generation } & \multirow{2}{*}{ Oviposition } & \multirow{2}{*}{$\begin{array}{c}\text { Post- } \\
\text { Oviposition }\end{array}$} & \multicolumn{2}{|c|}{ Longevity, mean $\pm \mathrm{SD}$} & \multicolumn{2}{|c|}{ Life span mean \pm SD } \\
\hline & & & & & q & $\hat{0}$ & q & $\hat{0}$ \\
\hline \multicolumn{9}{|l|}{ A. mangiferae } \\
\hline $25 \pm 1$ and $60 \%$ & $2.41 \pm 0.18$ & $12.28 \pm 0.57 \mathrm{a}$ & $17.75 \pm 0.40$ & $3.16 \pm 0.19$ & $23.32 \pm 0.35 \mathrm{a}$ & $22.15 \pm 0.27 \mathrm{a}$ & $33.19 \pm 0.29 \mathrm{a}$ & $30.52 \pm 0.90 \mathrm{a}$ \\
\hline $30 \pm 1$ and $55 \%$ & $2.00 \pm 0.11$ & $10.27 \pm 0.40 \mathrm{~b}$ & $16.6 \pm 0.51$ & $3.15 \pm 0.24$ & $21.75 \pm 0.45 b$ & $20.00 \pm 0.43 \mathrm{~b}$ & $30.00 \pm 0.20 \mathrm{~b}$ & $27.2 \pm 0.83 \mathrm{~b}$ \\
\hline $35 \pm 1$ and $50 \%$ & $1.63 \pm 0.07$ & $7.80 \pm 0.43 \mathrm{c}$ & $17.30 \pm 0.24$ & $2.60 \pm 0.23$ & $21.53 \pm 0.56 b$ & $17.75 \pm 0.53 \mathrm{c}$ & $27.73 \pm 0.43 c$ & $23.32 \pm 0.20 \mathrm{c}$ \\
\hline \multicolumn{9}{|l|}{ M. mangiferae } \\
\hline $25 \pm 1$ and $60 \%$ & $2.90 \pm 0.20$ & $12.50 \pm 0.24 \mathrm{a}$ & $17.20 \pm 0.23$ & $3.80 \pm 0.20$ & $23.90 \pm 0.47 \mathrm{a}$ & $22.20 \pm 0.43 \mathrm{a}$ & $33.50 \pm 0.35 \mathrm{a}$ & $31.20 \pm 0.85 \mathrm{a}$ \\
\hline $30 \pm 1$ and $55 \%$ & $2.08 \pm 0.23$ & $10.13 \pm 0.37 \mathrm{~b}$ & $15.58 \pm 0.24$ & $2.83 \pm 0.20$ & $20.50 \pm 0.33 b$ & $16.7 \pm 0.20 \mathrm{~b}$ & $28.50 \pm 0.47 \mathrm{~b}$ & $24.50 \pm 0.33 \mathrm{~b}$ \\
\hline $35 \pm 1$ and $50 \%$ & $1.41 \pm 0.09$ & $7.60 \pm 0.54 \mathrm{c}$ & $16.58 \pm 0.32$ & $2.16 \pm 0.24$ & $20.16 \pm 0.39 b$ & $17.77 \pm .24 \mathrm{c}$ & $26.35 \pm 0.20 \mathrm{c}$ & $23.67 \pm 0.36 \mathrm{~b}$ \\
\hline \multicolumn{9}{|l|}{ C. kenyae } \\
\hline $25 \pm 1$ and $60 \%$ & $3.30 \pm 0.08$ & $12.09 \pm 0.63 \mathrm{a}$ & $18.60 \pm 0.24$ & $4.20 \pm 0.31$ & $26.10 \pm 0.56 \mathrm{a}$ & $22.60 \pm 0.45 \mathrm{a}$ & $34.89 \pm 0.83 \mathrm{a}$ & $31.5 \pm 0.47 \mathrm{a}$ \\
\hline $30 \pm 1$ and $55 \%$ & $2.40 \pm 0.20$ & $10.55 \pm 0.20 \mathrm{~b}$ & $14.30 \pm 0.32$ & $2.80 \pm 0.24$ & $19.50 \pm 0.27 \mathrm{~b}$ & $17.42 \pm 0.33 b$ & $27.50 \pm 0.23 b$ & $24.70 \pm 0.56 \mathrm{~b}$ \\
\hline $35 \pm 1$ and $50 \%$ & $2.25 \pm 0.17$ & $8.64 \pm 0.20 \mathrm{c}$ & $16.90 \pm 0.24$ & $3.00 \pm 0.01$ & $22.16 \pm 0.23 b$ & $18.60 \pm 0.57 \mathrm{c}$ & $28.55 \pm 0.40 \mathrm{~b}$ & $24.48 \pm 0.63 b$ \\
\hline \multicolumn{9}{|l|}{ O. mangiferus } \\
\hline $25 \pm 1$ and $60 \%$ & $4.10 \pm 0.24$ & $13.49 \pm 0.24 \mathrm{a}$ & $16.80 \pm 0.53$ & $3.60 \pm 0.13$ & $24.5 \pm 0.35 \mathrm{a}$ & $23.60 \pm 0.39 \mathrm{a}$ & $33.90 \pm 0.95 \mathrm{a}$ & $32.0 \pm 1.12 \mathrm{a}$ \\
\hline $30 \pm 1$ and $55 \%$ & $2.58 \pm 0.20$ & $10.90 \pm 0.30 \mathrm{~b}$ & $12.60 \pm 0.49$ & $2.91 \pm 0.17$ & $18.10 \pm 0.53 \mathrm{~b}$ & $15.73 \pm 0.45 \mathrm{~b}$ & $26.50 \pm 0.59 b$ & $23.55 \pm 0.97 \mathrm{~b}$ \\
\hline $35 \pm 1$ and $50 \%$ & $2.10 \pm 0.31$ & $8.21 \pm 0.41 \mathrm{c}$ & $15.6 \pm 0.53$ & $2.40 \pm 0.09$ & $20.10 \pm 0.74 b$ & $18.63 \pm 0.24 c$ & $26.20 \pm 0.20 \mathrm{~b}$ & $24.33 \pm 0.83 b$ \\
\hline
\end{tabular}


Table (3): Female fecundity of Typhlodromips swirskii, fed on different mite prey species at different temperatures and relative humidities.

\begin{tabular}{ccccccccc}
\hline \multirow{2}{*}{$\begin{array}{c}\text { Temperature }\left({ }^{\circ} \mathrm{C}\right) \\
\&\end{array}$ R.H } & \multicolumn{3}{c}{ A. mangiferae } & \multicolumn{3}{c}{ M. mangiferae } & \multicolumn{3}{c}{ C. kenyae } & \multicolumn{3}{c}{ O. mangiferus } \\
\cline { 2 - 9 } & Mean \pm SD & Daily rate & Mean \pm SD & Daily rate & Mean \pm SD & Daily rate & Mean \pm SD & Daily rate \\
\hline $25^{\circ} \mathrm{C} \& 60 \%$ & $34.08 \pm 1.81^{\mathrm{a}}$ & 1.92 & $29.72 \pm 0.98^{\mathrm{a}}$ & 1.72 & $30.3 \pm 1.19^{\mathrm{a}}$ & 1.62 & $20.9 \pm 1.91^{\mathrm{a}}$ & 1.20 \\
\hline $30^{\circ} \mathrm{C} \& 55 \%$ & $35.69 \pm 1.72^{\mathrm{a}}$ & 2.15 & $32 \pm 1.16^{\mathrm{b}}$ & 2.60 & $33.1 \pm 1.15^{\mathrm{b}}$ & 2.31 & $20.50 \pm 2.70^{\mathrm{a}}$ & 1.62 \\
\hline $35^{\circ} \mathrm{C} \& 50 \%$ & $45.36 \pm 2.11^{\mathrm{b}}$ & 2.62 & $37.00 \pm 2.17^{\mathrm{c}}$ & 2.23 & $39.75 \pm 2.89^{\mathrm{c}}$ & 2.35 & $28.60 \pm 2.39^{\mathrm{b}}$ & 1.83 \\
\hline
\end{tabular}

Different letters in vertical columns denote a significant difference (F-test, $\mathrm{P}<0.05, \mathrm{P}<0.01)$

Table (4): Feeding capacity of Typhlodromips swirskii fed on different mite prey species at $25^{\circ} \mathrm{C}$ and $60 \%$ R.H.

\begin{tabular}{|c|c|c|c|c|c|c|c|c|c|}
\hline \multirow[b]{2}{*}{ Predator } & $\mathrm{S}$ & \multicolumn{8}{|c|}{ No. of consumed prey } \\
\hline & $\begin{array}{l}\mathrm{e} \\
\mathrm{x}\end{array}$ & $\begin{array}{c}A . \\
\text { mangiferae }\end{array}$ & $\begin{array}{c}\text { Daily } \\
\text { rate }\end{array}$ & $\begin{array}{c}M . \\
\text { mangiferae }\end{array}$ & $\begin{array}{c}\text { Daily } \\
\text { rate }\end{array}$ & $\begin{array}{c}C . \\
\text { Kenyae }\end{array}$ & $\begin{array}{c}\text { Daily } \\
\text { rate }\end{array}$ & $\begin{array}{c}O . \\
\text { mangiferus }\end{array}$ & $\begin{array}{c}\text { Daily } \\
\text { rate }\end{array}$ \\
\hline Protonymph & $\begin{array}{l}0 \\
+ \\
0\end{array}$ & $\begin{array}{c}44.91 \pm 1.17 \\
37.7 \pm 2.11\end{array}$ & $\begin{array}{l}20.79 \\
20.48\end{array}$ & $\begin{array}{l}24.9 \pm 0.87 \\
28.8 \pm 0.79\end{array}$ & $\begin{array}{l}11.42 \\
13.98\end{array}$ & $\begin{array}{l}34.8 \pm 0.97 \\
22.4 \pm 1.02\end{array}$ & 17.4 & $\begin{array}{c}8 \pm 0.66 \\
7.20 \pm 0.68\end{array}$ & $\begin{array}{l}4.00 \\
3.61\end{array}$ \\
\hline Deutonymph & $\begin{array}{l}\text { o } \\
\text { t } \\
0\end{array}$ & $\begin{array}{l}84.9 \pm 1.19 \\
67.2 \pm 1.47\end{array}$ & $\begin{array}{l}37.7 \\
36.5\end{array}$ & $\begin{array}{l}67.3 \pm 1.02 \\
69.8 \pm 0.97\end{array}$ & $\begin{array}{c}28.5 \\
31.75\end{array}$ & $\begin{array}{c}71.8 \pm 1.11 \\
53.4 \pm 2.3\end{array}$ & $\begin{array}{l}35.9 \\
26.7\end{array}$ & $\begin{array}{l}12.7 \pm 0.97 \\
13.1 \pm 0.24\end{array}$ & $\begin{array}{l}5.70 \\
6.35\end{array}$ \\
\hline Total & $\begin{array}{l}\text { p } \\
\text { + } \\
\text { ot }\end{array}$ & $\begin{array}{c}129.81 \pm 1.57^{\mathrm{a}} \\
104.9 \pm 2.14^{\mathrm{a}}\end{array}$ & $\begin{array}{l}29.43 \\
28.50\end{array}$ & $\begin{array}{l}92.2 \pm 1.93^{\mathrm{b}} \\
98.6 \pm 1.01^{\mathrm{a}}\end{array}$ & $\begin{array}{l}20.33 \\
23.14\end{array}$ & $\begin{array}{c}106.6 \pm 1.14^{\mathrm{c}} \\
75.8 \pm 2.13^{\mathrm{b}}\end{array}$ & $\begin{array}{l}26.65 \\
19.28\end{array}$ & $\begin{array}{l}20.70 \pm 1.02^{\mathrm{d}} \\
20.30 \pm 1.01^{\mathrm{c}}\end{array}$ & $\begin{array}{l}4.90 \\
4.89\end{array}$ \\
\hline Pre- ovipositiom & o & $254.5 \pm 3.11$ & 118.9 & $201.4 \pm 3.12$ & 69.4 & $234.5 \pm 3.1$ & 71.06 & $48 \pm 2.1$ & 11.70 \\
\hline Generation & 우 & $384.3 \pm 6.71^{\mathrm{a}}$ & 31.29 & $293.6 \pm 2.92^{\mathrm{b}}$ & 23.4 & $341.1 \pm 2.09^{c}$ & 28.21 & $68.70 \pm 1.98^{\mathrm{d}}$ & 5.09 \\
\hline Oviposition & 우 & $2058.7 \pm 85.31^{\mathrm{a}}$ & 115.98 & $1837.09 \pm 43.39^{\mathrm{b}}$ & 106.8 & $2175.4 \pm 4.1^{\mathrm{a}}$ & 116.9 & $233.1 \pm 8.12^{\mathrm{c}}$ & 13.87 \\
\hline Post- oviposition & q & $85.83 \pm 2.79$ & 27.16 & $123.2 \pm 2.19$ & 32.4 & $111.1 \pm 3.21$ & 26.4 & $21.50 \pm 2.1$ & 5.90 \\
\hline Longevity & $\begin{array}{l}0 \\
+ \\
0 \\
0\end{array}$ & $\begin{array}{c}2399.03 \pm 74.11^{\mathrm{a}} \\
1958.3 \pm 44.8^{\mathrm{a}}\end{array}$ & $\begin{array}{c}102.8 \\
88.4\end{array}$ & $\begin{array}{c}2161.69 \pm 39.15^{\mathrm{b}} \\
1804.3 \pm 44.18^{\mathrm{b}}\end{array}$ & $\begin{array}{l}90.44 \\
81.27\end{array}$ & $\begin{array}{c}2521 \pm 7731^{\mathrm{c}} \\
2112.5 \pm 48.01 \mathrm{c}\end{array}$ & $\begin{array}{l}96.59 \\
93.47\end{array}$ & $\begin{array}{l}302.60 \pm 7.19^{d} \\
268.80 \pm 6.13^{d}\end{array}$ & $\begin{array}{l}12.35 \\
11.38\end{array}$ \\
\hline Life span & $\begin{array}{l}0 \\
+ \\
0\end{array}$ & $\begin{array}{l}2528.8 \pm 66.71^{\mathrm{a}} \\
2063.2 \pm 57.11^{\mathrm{a}}\end{array}$ & $\begin{array}{l}76.16 \\
67.51\end{array}$ & $\begin{array}{c}2220.1 \pm 40.11^{\mathrm{b}} \\
1902.9 \pm 51.9^{\mathrm{b}}\end{array}$ & $\begin{array}{l}66.27 \\
60.99\end{array}$ & $\begin{array}{l}2627.6 \pm 53.01^{\mathrm{c}} \\
2188.3 \pm 93.11^{\mathrm{c}}\end{array}$ & $\begin{array}{c}75.3 \\
70.43\end{array}$ & $\begin{array}{l}323.3 \pm 8.71^{\mathrm{d}} \\
289.1 \pm 9.11^{\mathrm{d}}\end{array}$ & $\begin{array}{l}9.53 \\
8.86\end{array}$ \\
\hline
\end{tabular}

Mean \pm SD: Different letters in horizontal columns denote significant difference (F- test, $\mathrm{P}<0.01$ ).

Table (5): Feeding capacity of Typhlodromips swirskii fed on different mit prey species at $30^{\circ} \mathrm{C}$ and $55 \%$ R.H.

\begin{tabular}{|c|c|c|c|c|c|c|c|c|c|}
\hline \multirow[b]{2}{*}{ Predator } & \multirow{2}{*}{$\begin{array}{l}\mathrm{S} \\
\mathrm{e} \\
\mathrm{x}\end{array}$} & \multicolumn{7}{|c|}{ No. of consumed prey } & \multirow[b]{2}{*}{$\begin{array}{c}\text { Daily } \\
\text { rate }\end{array}$} \\
\hline & & $\begin{array}{c}\text { A. } \\
\text { mangiferae }\end{array}$ & $\begin{array}{l}\text { Daily } \\
\text { rate }\end{array}$ & $\begin{array}{c}M . \\
\text { mangiferae }\end{array}$ & $\begin{array}{l}\text { Daily } \\
\text { rate }\end{array}$ & $\begin{array}{c}C . \\
\text { Kenyae }\end{array}$ & $\begin{array}{l}\text { Daily } \\
\text { rate }\end{array}$ & $\begin{array}{c}O . \\
\text { mangiferus }\end{array}$ & \\
\hline Protonymph & $\begin{array}{l}+ \\
+ \\
0\end{array}$ & $\begin{array}{l}24.3 \pm 0.77 \\
23.9 \pm 0.63\end{array}$ & $\begin{array}{c}13.2 \\
14.59\end{array}$ & $\begin{array}{c}11.58 \pm 0.91 \\
15.1 \pm 0.87\end{array}$ & $\begin{array}{l}8.21 \\
8.96\end{array}$ & $\begin{array}{l}29.7 \pm 1.12 \\
18.2 \pm 1.23\end{array}$ & 16.5 & $\begin{array}{l}6.5 \pm 0.24 \\
8.2 \pm 0.33\end{array}$ & $\begin{array}{c}3.55 \\
4.9 \\
\end{array}$ \\
\hline Deutonymph & $\begin{array}{l}\text { क } \\
+ \\
0\end{array}$ & $\begin{array}{l}48.92 \pm 0.59 \\
37.92 \pm 1.12\end{array}$ & $\begin{array}{c}26.6 \\
28.09\end{array}$ & $\begin{array}{c}28.83 \pm 0.62 \\
30.8 \pm 1.2\end{array}$ & $\begin{array}{l}17.36 \\
18.22\end{array}$ & $\begin{array}{l}50.2 \pm 1.91 \\
37.7 \pm 3.11\end{array}$ & 26.4 & $\begin{array}{c}11.08 \pm 1.6 \\
12.06 \pm 0.97\end{array}$ & $\begin{array}{c}5.80 \\
6.2 \\
\end{array}$ \\
\hline Total & $\begin{array}{l}0 \\
+ \\
0 \\
0\end{array}$ & $\begin{array}{l}73.22 \pm 0.99 \mathrm{a} \\
61.82 \pm 1.29 \mathrm{a}\end{array}$ & $\begin{array}{l}19.89 \\
20.67\end{array}$ & $\begin{array}{c}40.41 \pm 1.07 b \\
45.9 \pm 1.19 b\end{array}$ & $\begin{array}{l}13.16 \\
13.90\end{array}$ & $\begin{array}{l}79.9 \pm 2.04 \mathrm{c} \\
55.9 \pm 3.19 \mathrm{c}\end{array}$ & $\begin{array}{l}19.97 \\
17.80\end{array}$ & $\begin{array}{l}17.58 \pm 1.77 \mathrm{~d} \\
20.26 \pm 1.88 \mathrm{~d}\end{array}$ & $\begin{array}{l}4.70 \\
5.64\end{array}$ \\
\hline Pre-ovipositiom & q & $141.07 \pm 2.13$ & 70.5 & $125.25 \pm 3.2$ & 60.21 & $164.3 \pm 3.15$ & 68.45 & $23.5 \pm 4.1$ & 9.1 \\
\hline Generation & 오 & $214.29 \pm 2.19 \mathrm{a}$ & 20.8 & $165.66 \pm 4.7 \mathrm{~b}$ & 16.08 & $244.2 \pm 2.97 \mathrm{c}$ & 23.1 & $41.08 \pm 3.12 \mathrm{~d}$ & 3.83 \\
\hline Oviposition & 오 & $2222.1 \pm 85.12 \mathrm{a}$ & 133.9 & $1624.3 \pm 29.81 b$ & 104.25 & $1964.6 \pm 53.79 \mathrm{c}$ & 137.3 & $168.08 \pm 11.13 \mathrm{~d}$ & 13.33 \\
\hline Post-oviposition & 운 & $97.3 \pm 9.11$ & 3.9 & $103.25 \pm 5.39$ & 36.48 & $106.6 \pm 11.06$ & 38.07 & $21.9 \pm 3.2$ & 7.53 \\
\hline Longevity & $\begin{array}{l}\text { क } \\
+ \\
0 \\
\end{array}$ & $\begin{array}{c}2460.47 \pm 75.21 \mathrm{a} \\
1908 \pm 27.1 \mathrm{a} \\
\end{array}$ & $\begin{array}{c}113.3 \\
95.4 \\
\end{array}$ & $\begin{array}{c}1852.8 \pm 54.80 \mathrm{~b} \\
14.64 \pm 53.19 \mathrm{~b} \\
\end{array}$ & $\begin{array}{c}90.38 \\
87.7 \\
\end{array}$ & $\begin{array}{l}2235.5 \pm 59.21 \mathrm{c} \\
1897.5 \pm 64.15 \mathrm{a}\end{array}$ & $\begin{array}{l}114.6 \\
108.9 \\
\end{array}$ & $\begin{array}{l}213.48 \pm 14.7 \mathrm{~d} \\
213.16 \pm 11.39 \\
\end{array}$ & $\begin{array}{l}11.79 \\
11.77 \\
\end{array}$ \\
\hline Life span & $\begin{array}{l}0 \\
+ \\
0\end{array}$ & $\begin{array}{l}2533.7 \pm 45.11 \mathrm{a} \\
1969.8 \pm 23.12 \mathrm{a}\end{array}$ & $\begin{array}{l}84.3 \\
72.4\end{array}$ & $\begin{array}{l}1898.7 \pm 66.71 b \\
1509.9 \pm 54.12 b\end{array}$ & $\begin{array}{l}66.52 \\
61.62 \\
\end{array}$ & $\begin{array}{l}2315.4 \pm 63.71 \mathrm{a} \\
1953.4 \pm 72.81 \mathrm{a}\end{array}$ & $\begin{array}{l}83.73 \\
79.08 \\
\end{array}$ & $\begin{array}{l}231.06 \pm 8.91 \mathrm{c} \\
233.3 \pm 12.17 \mathrm{c}\end{array}$ & $\begin{array}{c}8.71 \\
8.8 \\
\end{array}$ \\
\hline
\end{tabular}

Mean \pm SD: Different letters in horizontal columns denote significant difference $(F-$ test, $\mathrm{P}<0.01)$ 
Table (6): Feeding capacity of the Typhlodromips swirskii fed on different mite prey species at $35^{\circ} \mathrm{C}$ and $50 \%$ R.H.

\begin{tabular}{|c|c|c|c|c|c|c|c|c|c|}
\hline \multirow[b]{2}{*}{ Predator } & \multirow{2}{*}{$\begin{array}{l}\mathrm{S} \\
\mathrm{e} \\
\mathrm{x}\end{array}$} & \multicolumn{7}{|c|}{ No. of consumed prey } & \multirow[b]{2}{*}{$\begin{array}{l}\text { Daily } \\
\text { rate }\end{array}$} \\
\hline & & $\begin{array}{c}A . \\
\text { mangiferae }\end{array}$ & ily rate & $\begin{array}{c}M . \\
\text { mangiferae }\end{array}$ & $\begin{array}{c}\text { Daily } \\
\text { rate }\end{array}$ & C. Kenyae & ily rate & $\begin{array}{c}O . \\
\text { mangiferus }\end{array}$ & \\
\hline \multirow[t]{2}{*}{ Protonymph } & 우 & $15.36 \pm 0.8$ & 13.01 & $16.3 \pm 0.9$ & 15.1 & $16.58 \pm 1.21$ & 14.29 & $6.2 \pm 0.21$ & 5.6 \\
\hline & $\hat{0}$ & $9.25 \pm 0.97$ & 9.25 & $12.53 \pm 0.78$ & 12.53 & $9 \pm 0.92$ & 8.33 & $4.63 \pm 0.24$ & 4.63 \\
\hline \multirow{2}{*}{ Deutonymph } & 우 & $22.27 \pm 1.31$ & 19.23 & $18.9 \pm 1.2$ & 18.9 & $26.33 \pm 1.2$ & 21.06 & $7.9 \pm 0.04$ & 7.18 \\
\hline & $\hat{0}$ & $17 \pm 1.08$ & 17 & $19.53 \pm 1.3$ & 19.53 & $20.5 \pm 1.3$ & 18.98 & $6.09 \pm 0.07$ & 6.09 \\
\hline \multirow[t]{2}{*}{ Total } & 우 & $37.63 \pm 2.11$ & 15.94 & $35.2 \pm 2.01^{\mathrm{a}}$ & 16.92 & $42.91 \pm 2.42^{\mathrm{b}}$ & 17.80 & $14.1 \pm 0.88^{\mathrm{c}}$ & 6.40 \\
\hline & $\hat{0}$ & $26.25 \pm 1.92^{\mathrm{a}}$ & 13.12 & $32.06 \pm 1.09^{\mathrm{b}}$ & 16.03 & $29.5 \pm 2.91^{\mathrm{a}}$ & 13.65 & $10.72 \pm 0.9^{c}$ & 5.36 \\
\hline Pre- ovipositiom & o & $88.09 \pm 2.19$ & 54.04 & $71.2 \pm 3.11$ & 50.53 & $162.7 \pm 4.15$ & 72.3 & $23.1 \pm 1.11$ & 11 \\
\hline Generation & 오 & $125.72 \pm 3.61^{\mathrm{a}}$ & 16.11 & $1006.4 \pm 8.91^{b}$ & 14 & $205.6 \pm 3.19^{c}$ & 23.8 & $37.2 \pm 2.00^{\mathrm{d}}$ & 4.53 \\
\hline Oviposition & q & $2312.27 \pm 55.47^{\mathrm{a}}$ & 133.6 & $1744.66 \pm 67.1^{b}$ & 105.2 & $2237.9 \pm 87.11^{\mathrm{a}}$ & 132.4 & $258.4 \pm 3.91^{\mathrm{c}}$ & 16.56 \\
\hline Post-oviposition & 우 & $144.3 \pm 13.79$ & 55.52 & $88.4 \pm 2.31$ & 40.9 & $129.58 \pm 13.1$ & 43.19 & $11.6 \pm 0.9$ & 4.8 \\
\hline \multirow[t]{2}{*}{ Longevity } & q & $2544.6 \pm 64.17^{\mathrm{a}}$ & 117.8 & $1904.2 \pm 45.9^{b}$ & 94.4 & $2530.18 \pm 63.1^{\mathrm{a}}$ & 114.18 & $293.1 \pm 6.11^{\mathrm{c}}$ & 14.58 \\
\hline & $\hat{0}$ & $1885.2 \pm 52.19^{\mathrm{a}}$ & 106.2 & $1384.8 \pm 63.4^{\mathrm{b}}$ & 68.7 & $1751.9 \pm 64.2^{\mathrm{a}}$ & 94.19 & $218.3 \pm 8.7^{\mathrm{c}}$ & 11.72 \\
\hline \multirow[t]{2}{*}{ Life span } & o & $2582.3 \pm 43.91^{\mathrm{a}}$ & 92.88 & $1939.4 \pm 63.7^{b}$ & 73.6 & $2573.09 \pm 83.1^{\mathrm{a}}$ & 90.1 & $307.2 \pm 12.3^{\mathrm{c}}$ & 11.72 \\
\hline & o & $1911.45 \pm 45.19^{\mathrm{a}}$ & 81.9 & $1416.8 \pm 34.11^{\mathrm{b}}$ & 59.8 & $1781.4 \pm 71.1^{\mathrm{c}}$ & 72.76 & $229.02 \pm 9.12^{\mathrm{d}}$ & 9.41 \\
\hline
\end{tabular}

Mean \pm SD; Different letters in horizontal columns denote significant different (F- test, $\mathrm{P}<0.01$ ).

Table (7): Effect of different prey species on life table parameters of Typhlodromips swirskii at different temperatures and R.H.

\begin{tabular}{|c|c|c|c|c|c|c|c|c|c|c|c|c|}
\hline \multirow[b]{2}{*}{ Life table parameters } & \multicolumn{3}{|c|}{$25 \pm 1$ and $60 \%$} & \multicolumn{4}{|c|}{$30 \pm 1$ and $55 \%$} & \multicolumn{5}{|c|}{$35 \pm 1$ and $50 \%$} \\
\hline & 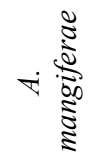 & 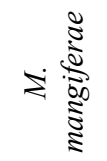 & ن & $0 . \frac{5}{5}$ & 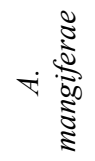 & 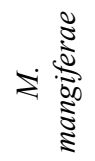 & ن & 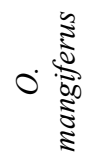 & 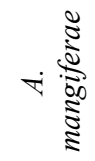 & 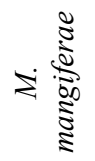 & ن & 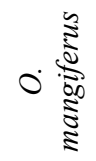 \\
\hline Net reproduction rate (Ro) & 23.82 & 19.58 & 22.26 & 14.62 & 24.94 & 22.51 & 23.1 & 14.34 & 32.81 & 25.71 & 27.47 & 18.47 \\
\hline Mean generation time (T.) & 20.1 & 20.52 & 21.20 & 20.14 & 18.31 & 17.9 & 17.29 & 17.05 & 16.16 & 16.01 & 16.92 & 15.87 \\
\hline Intrinsic rate of increase $(\mathrm{rm})$ & 0.157 & 0.144 & 0.146 & 0.133 & 0.175 & 0.173 & 0.181 & 0.156 & 0.216 & 0.20 & 0.195 & 0.183 \\
\hline Finite rate of increase $\left(\mathrm{e}^{\mathrm{rm}}\right)$ & 1.17 & 1.155 & 1.157 & 1.142 & 1.192 & 1.190 & 1.199 & 1.168 & 1.241 & 1.22 & 1.215 & 1.200 \\
\hline $50 \%$ mortality (in days) & 33 & 34 & 35 & 34 & 30 & 28 & 27 & 26 & 28 & 26 & 28 & 26 \\
\hline Sex ratio (Female/total) & $19 / 30$ & $19 / 30$ & $21 / 30$ & $19 / 30$ & $21 / 30$ & $21 / 30$ & $21 / 30$ & $21 / 30$ & $22 / 30$ & $21 / 30$ & $21 / 30$ & $19 / 30$ \\
\hline Sex ratio (female : male) & $2.3: 1$ & $1.72: 1$ & $2.3: 1$ & $1.72: 1$ & $2.3: 1$ & $2.3: 1$ & $2.3: 1$ & $2.3: 1$ & $2.75: 1$ & $2.3: 1$ & $2.3: 1$ & $1.72: 1$ \\
\hline
\end{tabular}

background about the prospects of using the phytoseiid predatory mite T. swirskii, as a biocontrol agent against the eriophyid mites infesting mango trees.

\section{REFERENCES}

Abou-Awad, B. A.; El-Sawaf, B. M. and AbdelKhalek, A. A. 1999. Impact of two eriophyid fig mites, Aceria ficus and Rhyncaphytoptus ficifoliae, as prey on postembryonic development and oviposition rate of the predacious mite Amblyseius swirskii. Acarologia, 40: 367-371.

Abou-Awad, B. A.; Metwally, A. M. and AlAzzazy, M. M. 2005. Environmental and biological aspects of two eriophyid olive mites in Egypt: Aceria oleae and Tegolophous hassani. Z. Pflanzenkrankh. Pflanzensch., 112: 287-303.

Abou-Awad, B. A.; Nasr, A. K.; Gomaa, E. A. and Abou-Elela, M. M. 1989. Life history of the predatory mite Cydrodromella negevi and the effect nutrition on its biology (Acari: Phytoseiidae). Insect. Sci. Appl., 10: 617-623.

Abou-Elella, G.M. 2003. Effect of eriophyid prey species and relative humidity on some biological aspects of the predatory mite, Proprioseiopsis (Amblyseius) lindiquisti( Acari: Phytoseiidae): Egypt. J. Biol. Pest Cont., 13: 31-33.

Al-Azzazy, M. M. 2005. Integrated management of mites infesting mango trees. Ph. D. Thesis, AlAzhar Univ., Fac. Agric.

Aly, F. S. 1994. Biological and ecological studies on some predacious mesostigmatic mites with special reference to the family Phytoseiidae. Ph. D. Thesis, Cairo Univ., Fac. Agric.

El-Laithy, A. Y. M. and Fouly, A. H. 1992. Life table parameters of the two phytoseiid predators Amblyseius scutalis (Athias-Henriot) and $A$. swirskii A.-H. (Acari: Phytoseiidae) in Egypt. J. App. Ent., 113: 8-12.

Hulting, F. L., Orr. D. B. and Obrycki, J. J. 1990. A computer program for calculation and statistical 
comparison of intrinsic rates of increase and associated life table parameters. Florida Entomol., 73: 601-612.

Mangini, A. C. Jr. and Hain, F. P. 1991. Vapor pressure deficit differentially affects laboratory populations of Metaseiulus occidentalis and Neoseiulus fallacies (Acarina: Phytoseiidae) reared together. Environ. Entomol., 20: 823-831.

Metwally, A. M.; Abou- Elnaga, M. M., Taha, H. A. and Hoda, F. M. 1984. Studies on feeding, reproduction and development of Amblyseius swirskii A.-H. (Acarina: Phytoseiidae). Agric. Res. Rev. , 62: 233-236.

Momen, F. M. 1999. Biological studies of Amblyseius lindquisti, a specific predator of eriophyid mites (Acari: Phytoseiidae: Eriophyidae). Acta Phytopathol. Entomol. Hung., 34: 245-251.

Momen, F. M. and El-Sawi, S. A. 1993. Biology and feeding behaviour of the predatory mite Amblyseius swirskii (Acarina: Phytoseiidae). Acarologia, 34:199-204.

Ragusa, S. and Swirski, E. 1976. Feeding habits, postembryonic and adult survival, mating, virility and fecundity of the predacious mite Amblyseius swirskii (Acarina: Phytoseiidae) on some coccids and mealy bugs. Entomophaga, 22: 383-392.

Rasmy, A. H.; Momen, F. M. and Zaher, M. A. 2002. Dietry influence on life history and predation of the phytoseiid mite Amblyseius deleoni. F. Bernini, R. Nonnelle, (eds) Acarid Phylogeny and Evolution: 319-323.

Sabelis, M. W. 1985. Reproduction. In: Helle, W. and Sabelis, M.W. (eds.): Spider mites: Their biology, natural enemies and control, 73-82. World Crop. Pests, Vol. 1 B, Elsevier, Amsterdam.

Swirski, E.; Amitai, S. and Dorzia, N. 1967. Laboratory studies on the feeding, development and reproduction of the predacious mites Amblyseius rubini Swirski and Amitai and Amblyseius swirskii Athias- Henriot (Acarina: Phytoseiidae) on various kinds of food substances. Israel J. Agric. Res., 17: 101-119.

Van Dinh, N.; Sabelis, M.W. and Janssen, A., 1988. The influence of humidity and water availability on the survival of Amblyseius idaeus and Amblyseius anonymous (Acarina: Phytoseiidae). Exp. Appl. Acarol., 4: 27-40.

Yousef, A. A. and Shehata, K. K. 1971. Mites associated with pome fruits trees in URA. Z. Ang. Entomol ., 67: 360-370. 